\title{
DIVERSIDAD DE FITOPLANCTON COMO INDICADOR DE CALIDAD DE AGUA EN LA CUENCA BAJA DEL RÍO LURÍN, LIMA, PERÚ
}

\section{PHYTOPLANKTON DIVERSITY AS AN INDICATOR OF WATER QUALITY IN THE LOWER BASIN OF THE LURIN RIVER, LIMA, PERU}

\author{
Armando Vélez-Azañero ${ }^{1}$, Sebastián Lozano ${ }^{1}$ y Keytel Cáceres-Torres ${ }^{1}$
}

\begin{abstract}
Resumen
Se establecieron seis sitios de muestreo (P) entre los 5 m.s.n.m. (P1) y 31 m.s.n.m. (P6) en la cuenca baja del río Lurín, Lima, Perú en agosto de 2008, para evaluar las características físicas y químicas del agua, y determinar su calidad en base a la diversidad de microalgas y cianobacterias presentes. Se registraron cinco especies de cianobacterias y 89 especies de microalgas distribuidas en cuatro divisiones, donde Bacillariophyta fue la más abundante con $72 \%$ del total de especies. El 31.2\% de las especies identificadas correspondió a los géneros Navicula, Fragilaria, Nitzschia y Synedra. Un total de 29 géneros y 55 especies fueron identificados exclusivamente en uno de los sitios. Se evidenció una disminución gradual de la similitud desde las dos estaciones más alejadas de la desembocadura (39.3\%) hasta las dos más cercanas (18.2\%). La mayor conductividad evaluada se presentó a los 31 m.s.n.m. con $1820 \mu \mathrm{S} . \mathrm{cm}^{-1}$ y la mínima a los 5 m.s.n.m. con 1390 $\mu \mathrm{S} . \mathrm{cm}^{-1}$. Los valores más bajos de $\mathrm{pH}$ fueron registrados en los sitios más altos (6.2; 6.1), y el valor máximo a 8 m.s.n.m. (8.2) presentándose así una correlación inversa entre ambas variables. La temperatura máxima del agua fue de $23^{\circ} \mathrm{C}$, y la mínima fue de $16^{\circ} \mathrm{C}$ guardando una relación directa con la altitud. El aumento de los niveles de recambio con la distancia espacial entre los sitios de muestreo deja clara la sensibilidad de las microalgas y cianobacterias a los cambios ambientales. Estos cambios se reflejan en una alta mineralización del sistema y bajos niveles de $\mathrm{pH}$ en el agua, y se deben principalmente a causas antropogénicas.

Palabras clave: Abundancia, Bacillariophyta, Cyanobacteria, microalgas, río Lurín, sensibilidad, similitud.
\end{abstract}

\begin{abstract}
Six sampling points (P) were established from 5 to 31 meters above sea level in the lower basin of the Lurin River, in august 2008, to evaluate the physical and chemical characteristics of water, and to determine how water quality relates to diversity of microalgae and Cyanobacteria. Five species of Cyanobacteria were recorded and 89 species of microalgae from four divisions were encountered. Bacillariophyta was the most abundant division and accounted for $72 \%$ of all species. The genera Navicula, Fragilaria, Nitzschia and Synedra accounted for $31.2 \%$ of the total identified species. A total of 29 genera and 55 species were identified exclusively from one of the sampling points. A gradual decrease of the similarity from the two stations with higher altitude (39.3\%) to the two lower stations (18.2\%) was evident. The highest conductivity value was recorded at 31 meters above sea level $\left(1820 \mu \mathrm{S} . \mathrm{cm}^{-1}\right)$ and the lowest at 5 meters above sea level $\left(1390 \mu \mathrm{S} . \mathrm{cm}^{-1}\right)$. The lowest $\mathrm{pH}$ values were registered at higher elevations $(6.2 ; 6.1)$, and the maximum value at 8 meters above sea level (8.2); this shows an inverse correlation between both variables. Temperature values ranged from $23^{\circ} \mathrm{C}$ to $16^{\circ} \mathrm{C}$ and it was directly related to altitude. Replacement levels, which increase with increasing spatial distance between sampling points, clearly showed the sensitivity of microalgae and Cyanobacteria to environmental changes. These changes are reflected in a high mineralization and low pH levels in the water, and are mainly due to natural and anthropogenic causes.
\end{abstract}

Key words: Abundance, Bacillariophyta, Cyanobacteria, Lurin river, microalgae, sensitivity, similarity.

\section{Introducción.}

En la cuenca baja del río Lurín, la agricultura, la ganadería y la presencia de asentamientos humanos están desencadenando diversas alteraciones en los ecosistemas terrestres y acuáticos, afectando así la presencia de organismos que brindan servicios 
ecosistémicos (Vélez-Azañero \& LizárragaTravaglini, 2013). Las modificaciones físicas y químicas en cursos de agua repercuten en las respuestas individuales, poblacionales, comunitarias y ecosistémicas de los organismos (Pinilla, 1998), entre los que destacan las microalgas, cianobacterias e insectos (empleados para monitorear la calidad del agua en ecosistemas lóticos; Iannacone et al., 2000). Por estas razones, se han convertido en un referente del estado ecológico de cualquier ecosistema acuático (Peña et al., 2005; Wan Maznah, 2010; Kshirsagar, 2013). En este sentido, se han realizado diversas e importantes investigaciones sobre la diversidad de comunidades zooplactónicas y fitoplanctónicas y su relación con la calidad del agua en ambientes lóticos de gran importancia tales como el río Lurín, el río Chillón y el río Rímac (Iannacone et al., 2013; Ruiz et al., 2007; Alvariño \& Iannacone, 2007; Yucra \& Tapia, 2008).

Las microalgas y cianobacterias como indicadoras son importantes en ambientes continentales acuáticos (Lobo et al., 1995; Licursi \& Gómez, 2003; Lobo et al., 2004; Medina et al., 2012) por su tolerancia y sensibilidad a los cambios ambientales, y por sus ciclos biológicos reducidos (Meybeck et al., 1992; Roset et al., 2001). El uso de microalgas y cianobacterias simplifica en gran medida las actividades de campo y laboratorio debido a que su aplicación solo requiere de la identificación y cuantificación de los organismos basándose en índices de diversidad (Vázquez et al., 2006).

El objetivo del trabajo fue determinar la diversidad de fitoplancton como indicador de calidad de agua en la cuenca baja del río Lurín, Lima, Perú.

\section{Materiales y métodos.}

Área de estudio.

Está ubicada en el distrito de Lurín, provincia de Lima, y forma parte de la cuenca baja del río Lurín (12¹1'27.12” y 12¹8'S; 7656' y 7650’43.2”O; Coral, 2007) con los distritos de Pachacamac y Cieneguilla (Alfaro \& Claverías, 2010). La cuenca del río Lurín es una de las 62 cuencas del Pacífico con una extensión superficial de 1 658.19km² (Ministerio de Agricultura del Perú, 2004). Está comprendida en las provincias de Lima y Huarochirí (Felipe-Morales, 2012) y conforma el hinterland de la capital de la República del Perú, junto al río Chillón y al río Rímac (Alfaro \& Claverías, 2010); este río está conectado con 10 distritos a lo largo de la parte alta, media y baja de la cuenca (Vega, 2010; Alfaro \& Claverías, 2010). Muestreo y Análisis.

Se establecieron seis sitios de muestreo (P1, P2, P3, P4, P5 y P6) entre los 5 m.s.n.m. y 31 m.s.n.m. (Tabla 1, Figura 1) en agosto de 2008. En cada sitio se colectó una muestra de agua en el margen derecho del río (mayor accesibilidad) y a 1 metro de la orilla, las que se conservaron en frascos pastilleros de $200 \mathrm{ml}$ con solución FAA (formaldehído: etanol: ácido acético: agua, 10:50:5:35; Galati et al., 2007). Las muestras se trasladaron al laboratorio de Ecología y Biodiversidad Animal de la Escuela Profesional de Biología, Facultad de Ciencias Naturales y Matemática, Universidad Nacional Federico Villarreal (EPB-FCCNM-UNFV), para su análisis. Para el reconocimiento de las diatomeas se realizó una limpieza previa de frústulos con $1 \mathrm{ml}$ de ácido clorhídrico $1 \mathrm{M}$ en tubos de ensayo con tapa hermética y se almacenaron en frascos de alcohol al 30\%. La identificación se realizó siguiendo las claves taxonómicas de Acleto \& Zúñiga (1998) y Bicudo \& Menezes (2006) para las microalgas, y Komárek \& Anagnostidis (1998, 2005) para las cianobacterias. Para la revisión de los nombres taxonómicos y las sinonimias se consultaron las bases de datos en línea: National Center for Biotechnology Information (NCBI) y AlgaeBase. En cada sitio de muestreo se determinó la profundidad media del cauce; asimismo, se evaluó $\mathrm{pH}$, conductividad eléctrica y temperatura mediante un multiparámetro portátil HANNA HI 98128.

Se calculó una matriz de distancias en función al índice cualitativo de Jaccard a partir de la cual se generó un dendrograma utilizando el método UPGMA (Unweighted Pair Group Method with Arithmetic Mean) (Borcard et al., 2011), y se determinó el coeficiente de correlación de Pearson (r) entre los parámetros físicos y químicos evaluados, mediante el software estadístico R Project versión 3.0.0. Además, se utilizó el software ArcGIS versión 10.2.2 para generar el mapa del área de estudio a partir del registro fotográfico obtenido de Google Earth 2015 (Figura 1).

\section{Resultados.}

Se registraron cinco especies de cianobacterias distribuidas en cinco géneros, y 89 especies de microalgas distribuidas en cuatro divisiones: Bacillariophyta (32 géneros y 66 especies), Chlorophyta (13 géneros y 18 especies), Euglenophyta (3 géneros y 4 especies) y Xanthophyta (1 género y 1 especie) (Tabla 2). La división Bacillariophyta presentó la mayor riqueza específica con un $72 \%$ del total de especies, siendo la clase Bacillariophyceae la de mayor aporte a este porcentaje. El 31.2\% de especies identificadas correspondió a los géneros Navicula, Fragilaria, Nitzschia y Synedra (Tabla 2). P5 presentó la mayor riqueza específica (43), seguido por P2, P3 y P6 que registraron 22 especies cada uno. P1 fue el sitio con menor presencia de especies (17; Tabla 2).

P5 presentó exclusivamente seis de las ocho especies del género Navicula y P2 seis de las siete especies del género Synedra. Schroderella sp., Stephanodiscus astraea y Scenedesmus sp. fueron las especies más frecuentes (presentes en cuatro sitios de 


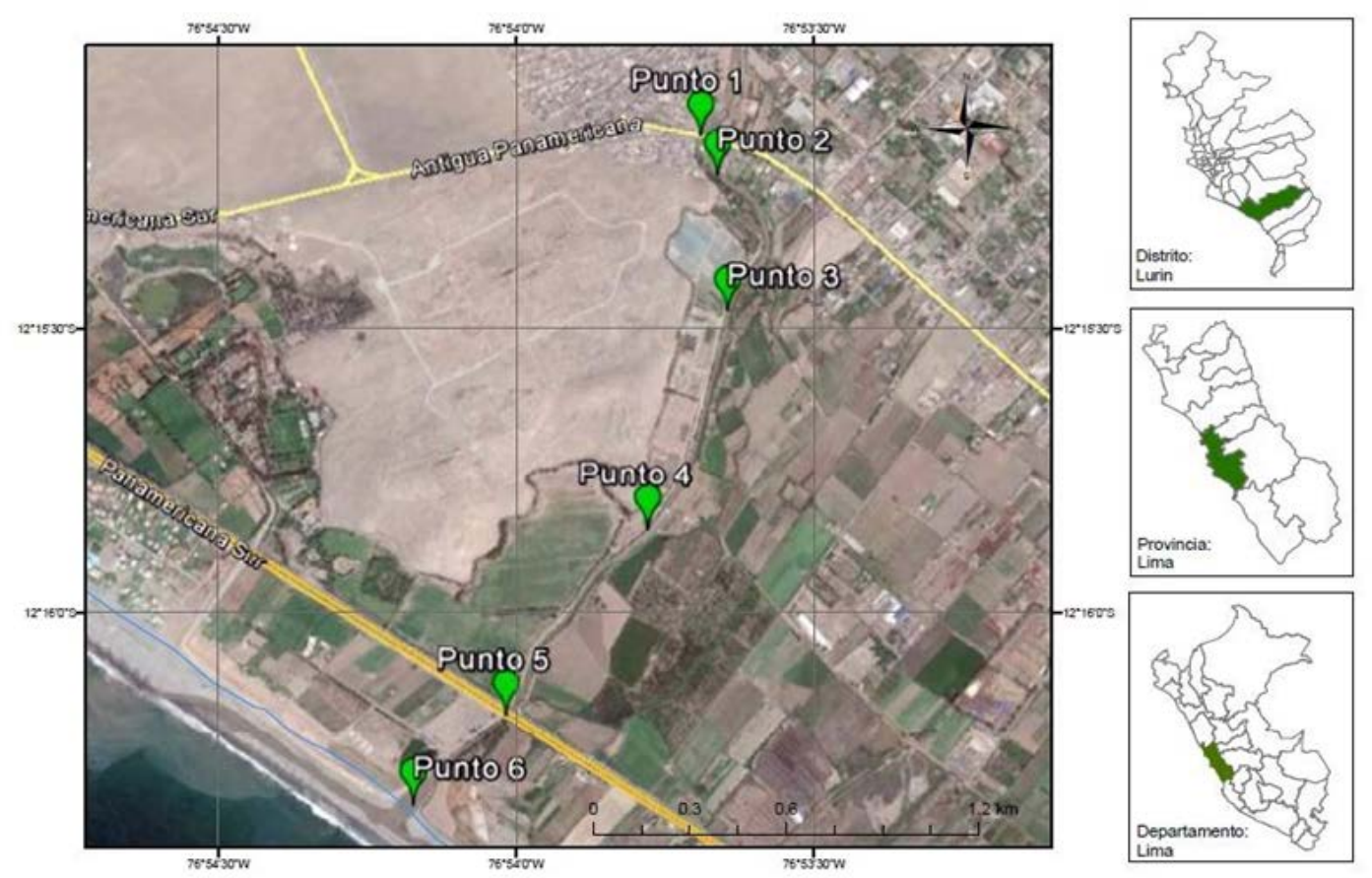

Figura 1. Mapa de la cuenca baja del río Lurín, Lima, Perú, donde se indican seis sitios de muestreo utilizados para la colecta de fitoplancton (Fuente: Google Earth 2015).

muestreo), mientras que un total de 29 géneros y 55 especies fueron identificados exclusivamente en uno de los sitios (P1: 3 géneros y 5 especies, P2: 3 géneros y 6 especies, P3: 4 géneros y 6 especies, P4: 2 géneros y 2 especies, P5: 12 géneros y 26 especies, P6: 5 géneros y 10 especies; Tabla 2).

En base a la matriz de distancias (índice de Jaccard y método UPGMA), se observó una mayor similitud entre P1 y P2 (39.3\%), mientras que los menores niveles de similitud se presentaron entre P1 y P6 (2.6\%), y entre P1 y P4 (2.8\%). Se evidenció una disminución gradual de similitud desde P1-P2 (39.3\%) hasta P5-P6 (18.2\%; Tabla 3, Figura 3).
Con respecto a los parámetros físicos y químicos, la mayor conductividad se registró en P6 con 1

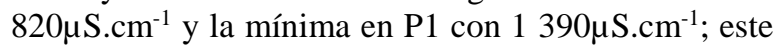
parámetro mostró una relación inversa moderada con la altitud (r: -0,47). Los valores más bajos de $\mathrm{pH}$ fueron registrados en P1 (6.2) y P2 (6.1), y el valor máximo en P5 (8.2). El pH se incrementó a medida que disminuyó la altitud en dirección a la desembocadura (r: -0.90). La temperatura máxima fue registrada en $\mathrm{P} 1\left(23^{\circ} \mathrm{C}\right)$, y la temperatura mínima en P6 $\left(16^{\circ} \mathrm{C}\right)$, con una variación total de $7^{\circ} \mathrm{C}$ (Tabla 4). La temperatura disminuyó gradualmente desde P1 hasta P6, presentando una relación directa muy alta

Tabla 1. Características y descripción de seis sitios de muestreo en la cuenca baja del río Lurín, Lima, Perú, en agosto de 2008.

\begin{tabular}{|c|c|c|c|}
\hline $\begin{array}{c}\text { Sitio de } \\
\text { muestreo }\end{array}$ & $\begin{array}{c}\text { Altitud } \\
\text { (m.s.n.m.) }\end{array}$ & $\begin{array}{l}\text { Coordenadas } \\
\text { geográficas }\end{array}$ & Descripción \\
\hline Sitio 1 (P1) & 31 & $\begin{array}{l}12^{\circ} 15^{\prime} 09.85^{\prime \prime} \mathrm{S} \\
76^{\circ} 53^{\prime} 41.37^{\prime \prime} \mathrm{O}\end{array}$ & $\begin{array}{l}\text { Asociado a vegetación herbácea. Influencia directa de la antigua } \\
\text { Panamericana Sur. Presencia moderada de residuos sólidos y desagües } \\
\text { domésticos. }\end{array}$ \\
\hline Sitio 2 (P2) & 25 & $\begin{array}{l}12^{\circ} 15 ’ 15.62 ” S \\
76^{\circ} 53 ’ 39.96 ” O\end{array}$ & $\begin{array}{l}\text { Asociado a campos de cultivo tradicional (tomate y maíz) e } \\
\text { hidropónico (tomate). Presencia considerable de residuos sólidos. } \\
\text { Asociado a una caballeriza. Influencia directa del proyecto "MESÍAS" }\end{array}$ \\
\hline Sitio 3 (P3) & 17 & $\begin{array}{l}12^{\circ} 15^{\prime} 28.06^{\prime \prime} \mathrm{S} \\
76^{\circ} 533^{\prime} 38.64 ” O\end{array}$ & $\begin{array}{l}\text { - SEDAPAL (aguas residuales; Figura 2). Aledaño a la planta de } \\
\text { tratamiento de aguas residuales (PTAR) - SEDAPAL. Vegetación } \\
\text { arbustiva y presencia moderada de residuos sólidos. }\end{array}$ \\
\hline Sitio 4 (P4) & 12 & $\begin{array}{l}12^{\circ} 15^{\prime} 51.26^{\prime \prime S} \\
76^{\circ} 53 ’ 46.10^{\prime \prime} \mathrm{O}\end{array}$ & $\begin{array}{l}\text { Asociado a campos de cultivo tradicional (acelga y coliflor). } \\
\text { Vegetación arbustiva y herbácea. }\end{array}$ \\
\hline Sitio 5 (P5) & 8 & $\begin{array}{l}12^{\circ} 16^{\prime} 11.48 ” \mathrm{~S} \\
76^{\circ} 54^{\prime} 00.94 ” \mathrm{O}\end{array}$ & $\begin{array}{l}\text { Influencia directa de la carretera Panamericana Sur. Presencia } \\
\text { moderada de residuos sólidos. }\end{array}$ \\
\hline Sitio 6 (P6) & 5 & $\begin{array}{l}12^{\circ} 16^{\prime} 20.02^{\prime \prime} \mathrm{S} \\
76^{\circ} 54^{\prime} 10.33^{\prime \prime} \mathrm{O}\end{array}$ & $\begin{array}{l}\text { Asociado a vegetación herbácea. Playa de piedras contaminada con } \\
\text { fragmentos de vidrios y metales. Sitio más cercano a la desembocadura } \\
\text { del río. }\end{array}$ \\
\hline
\end{tabular}




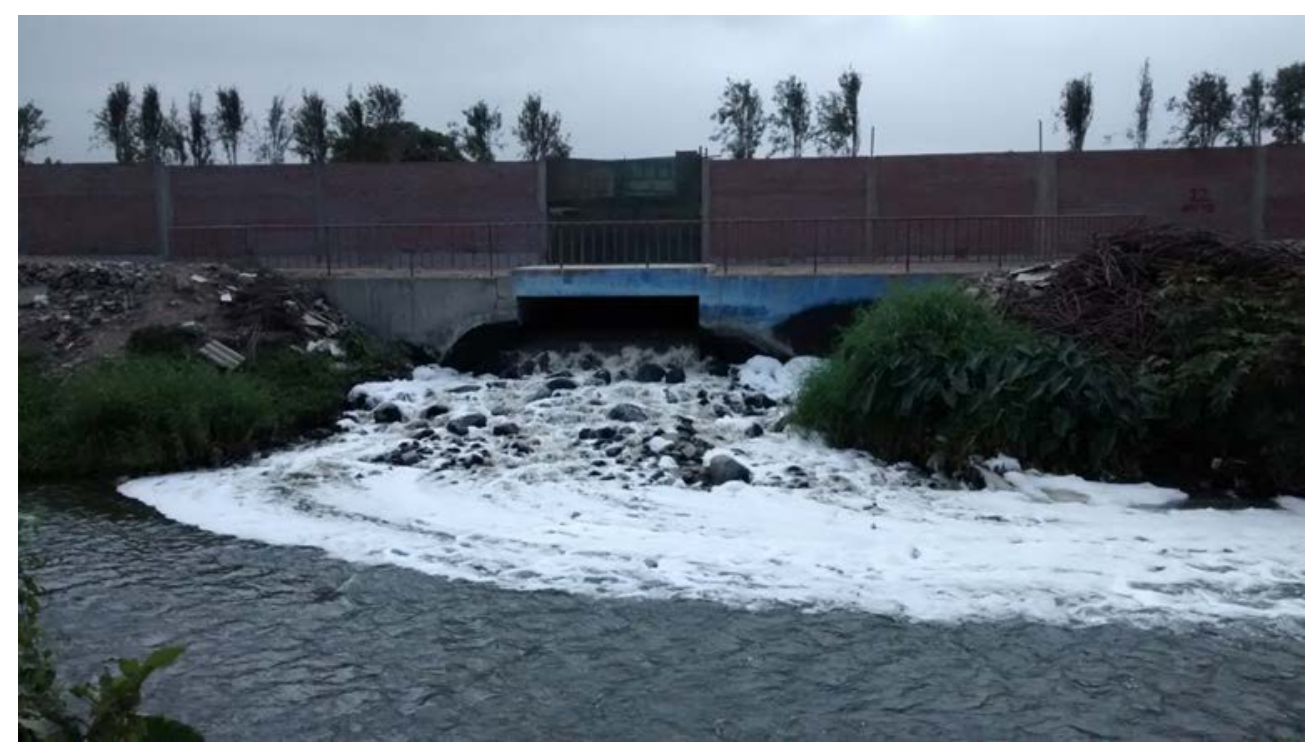

Figura 2. Vista del sitio de muestreo 3, donde hay una descarga directa del efluente del proyecto “MESÍAS” - SEDAPAL en la cuenca baja del río Lurín, Lima, Perú.

con la altitud (r: 0.88). Se determinó una correlación inversa alta entre la CE y la temperatura (r: -0.77), y entre el $\mathrm{pH}$ y la temperatura (r: -0.74). La profundidad media del cauce fue cambiando en los diferentes sitios, llegando a un mínimo de 12cm en P1 y P5, y un máximo de 66cm en P6 (Tabla 4).

Tabla 2: Especies de microalgas y cianobacterias identificadas en seis sitios de muestreo de la cuenca baja del río Lurín, Lima, Perú, en agosto de 2008.

\begin{tabular}{ccccccc}
\hline & \multicolumn{5}{c}{ Sitios de muestreo } \\
\cline { 3 - 6 } Especies & P1 & P2 & P3 & P4 & P5 & P6 \\
\hline
\end{tabular}

\section{CYANOBACTERIA}

Chroococcus sp.

Gloeocapsa sp.

Gloeotrichia sp.

Oscillatoria sp.

Tolypothrix lanata Wartmann ex Bornet \& Flahault

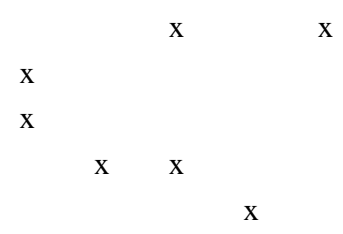

\section{MICROALGAS}

\section{DIVISIÓN: BACILLARIOPHYTA}

Clase: Bacillariophyceae

Achnanthes sp.

$\mathrm{X}$

Achnanthidium minutissimum (Kützing) Czarnecki

Amphora sp.

Berkeleya rutilans (Trentepohl ex Roth) Grunow

Berkeleya sp.

Caloneis sp.

Cocconeis placentula Ehrenberg

Cymbella affinis Kützing

Cymbella sp.

Diatoma vulgaris Bory de Saint-Vincent

Didymosphenia sp.

Epithemia sorex Kützing
$\mathrm{X}$

$\mathrm{X}$

$\mathrm{X}$

$\mathrm{X}$

$\mathrm{X}$

$\mathrm{X}$

$\mathrm{X}$

$\mathrm{X}$

$\mathrm{X}$

X X 
Tabla 2: Especies de microalgas y cianobacterias identificadas en seis sitios de muestreo de la cuenca baja del río Lurín, Lima, Perú, en agosto de 2008.

\begin{tabular}{|c|c|c|c|c|c|c|}
\hline \multirow[b]{2}{*}{ Especies } & \multicolumn{6}{|c|}{ Sitios de muestreo } \\
\hline & P1 & $\mathbf{P 2}$ & P3 & $\mathbf{P 4}$ & P5 & P6 \\
\hline Epithemia sp. & & & & $\mathrm{x}$ & $\mathrm{x}$ & \\
\hline Frustulia vulgaris (Thwaites) De Toni & & & $\mathrm{x}$ & $\mathrm{x}$ & & $\mathrm{x}$ \\
\hline Gomphonema parvulum (Kützing) Kützing & & & & & & $\mathrm{x}$ \\
\hline Gomphonema tergestinum (Grunow) Fricke & & & & & $\mathrm{x}$ & $\mathrm{x}$ \\
\hline Gomphonema sp. & & & $\mathrm{x}$ & & $\mathrm{x}$ & \\
\hline Hantzschia sp.1 & $\mathrm{x}$ & $\mathrm{x}$ & & & & \\
\hline Hantzschia sp.2 & $\mathrm{x}$ & & & & & \\
\hline Mastogloia elliptica (C.Agardh) Cleve & & & & $\mathrm{x}$ & & \\
\hline Meridion sp. & & & & & $\mathrm{x}$ & \\
\hline Navicula accomoda Hustedt & & & & & $\mathrm{x}$ & \\
\hline Navicula cryptocephala Kützing & & & & & $\mathrm{x}$ & \\
\hline Navicula cryptotenella Lange-Bertalot & & & & & $\mathrm{x}$ & \\
\hline Navicula reinhardtii (Grunow) Grunow & & & & & $\mathrm{x}$ & \\
\hline Navicula schroeteri Meister & & & & & $\mathrm{x}$ & \\
\hline Navicula subalpina Reichardt & & & & & $\mathrm{x}$ & \\
\hline Navicula tripunctata (O.F.Müller) Bory de Saint-Vincent & & & $\mathrm{x}$ & $\mathrm{x}$ & $\mathrm{x}$ & \\
\hline Navicula sp. & & & & & & $\mathrm{x}$ \\
\hline Nitzschia fonticola (Grunow) Grunow & & & & & $\mathrm{x}$ & \\
\hline Nitzschia gracilis Hantzsch & & & $\mathrm{x}$ & & & \\
\hline Nitzschia palea (Kützing) W.Smith & & & & & $\mathrm{x}$ & $\mathrm{x}$ \\
\hline Nitzschia scalaris (Ehrenberg) W.Smith & & & & & $\mathrm{x}$ & \\
\hline Nitzschia sp.1 & & $\mathrm{x}$ & & & & \\
\hline Nitzschia sp.2 & $\mathrm{x}$ & $\mathrm{x}$ & & & & \\
\hline Nitzschia sp.3 & & $\mathrm{x}$ & & & & \\
\hline Pinnularia sp.1 & & $\mathrm{x}$ & $\mathrm{x}$ & $\mathrm{x}$ & & \\
\hline Pinnularia sp.2 & $\mathrm{x}$ & & & & & \\
\hline Pinnularia sp.3 & $\mathrm{x}$ & $\mathrm{x}$ & & & & \\
\hline Placoneis pseudanglica E.J.Cox & & & & $\mathrm{x}$ & $\mathrm{x}$ & \\
\hline Rhopalodia sp. & $\mathrm{x}$ & & & & & \\
\hline Stauroneis anceps Ehrenberg & & & & & $\mathrm{x}$ & \\
\hline Surirella angustata Kützing & & & & & & $\mathrm{x}$ \\
\hline Synedra ulna (Nitzsch) Ehrenberg & & & & & & $\mathrm{x}$ \\
\hline Synedra sp.1 & & $\mathrm{x}$ & & $\mathrm{x}$ & & \\
\hline Synedra sp.2 & $\mathrm{x}$ & $\mathrm{x}$ & & & & \\
\hline Synedra sp.3 & & $\mathrm{x}$ & $\mathrm{x}$ & & & \\
\hline Synedra sp.4 & & $\mathrm{x}$ & $\mathrm{x}$ & & & \\
\hline Synedra sp.5 & $\mathrm{x}$ & $\mathrm{x}$ & & & & \\
\hline Synedra sp.6 & & $\mathrm{x}$ & & & & \\
\hline
\end{tabular}

Clase: Coscinodiscophyceae

Aulacoseira distans (Ehrenberg) Simonsen

Melosira varians C.Agardh

Skeletonema sp.

Clase: Fragilariophyceae

Fragilaria acus (Kützing) Lange-Bertalot

Fragilaria capucina Desmazières

Fragilaria capucina var. vaucheriae (Kützing) Lange-Bertalot

Fragilaria crotonensis Kitton

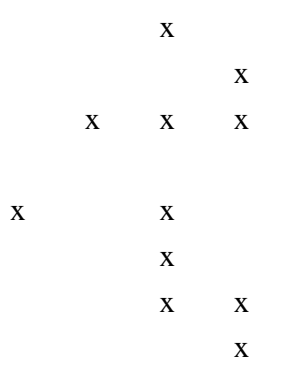


Tabla 2: Especies de microalgas y cianobacterias identificadas en seis sitios de muestreo de la cuenca baja del río Lurín, Lima, Perú, en agosto de 2008.

\begin{tabular}{|c|c|c|c|c|c|c|}
\hline \multirow[b]{2}{*}{ Especies } & \multicolumn{6}{|c|}{ Sitios de muestreo } \\
\hline & P1 & $\mathbf{P} 2$ & P3 & P4 & P5 & P6 \\
\hline Fragilaria sp.1 & $\mathrm{x}$ & & & & $\mathrm{x}$ & \\
\hline Fragilaria sp.2 & $\mathrm{x}$ & $\mathrm{x}$ & & & & \\
\hline Fragilaria sp.3 & & & & $\mathrm{x}$ & $\mathrm{x}$ & $\mathrm{x}$ \\
\hline Licmophora abbreviata C.Agardh & & & & $\mathrm{x}$ & & \\
\hline Thalassiothrix sp. & $\mathrm{x}$ & & & & & \\
\hline \multicolumn{7}{|l|}{ Clase: Mediophyceae } \\
\hline Cyclotella sp. & & & & & & $\mathrm{x}$ \\
\hline Schroederella delicatula (Peragallo) Pavillard & & & & $\mathrm{x}$ & $\mathrm{x}$ & $\mathrm{x}$ \\
\hline Schroederella sp. & $\mathrm{x}$ & $\mathrm{x}$ & & & $\mathrm{x}$ & $\mathrm{x}$ \\
\hline Stephanodiscus astraea (Ehrenberg) Grunow & & & $\mathrm{x}$ & $\mathrm{x}$ & $\mathrm{x}$ & $\mathrm{x}$ \\
\hline \multicolumn{7}{|l|}{ DIVISIÓN: CHLOROPHYTA } \\
\hline Acutodesmus incrassatulus (Bohlin) Tsarenko & & & & & $\mathrm{x}$ & \\
\hline Chlamydomonas reinhardtii Dangeard & & & & & $\mathrm{x}$ & \\
\hline Chlamydomonas sp. & & & $\mathrm{x}$ & & $\mathrm{x}$ & \\
\hline Cladophora sp. & $\mathrm{x}$ & $\mathrm{x}$ & & & & \\
\hline Coelastrum astroideum De Notaris & & & & & $\mathrm{x}$ & \\
\hline Coelastrum microporum Nägeli & & & & $\mathrm{x}$ & $\mathrm{x}$ & \\
\hline Cosmarium botrytis Meneghini ex Ralfs & & & $\mathrm{x}$ & $\mathrm{x}$ & & \\
\hline Keratococcus rhaphidioides (Hansgirg) Pascher & & & & & $\mathrm{x}$ & \\
\hline Pandorina sp. & $\mathrm{x}$ & & & & & \\
\hline Pediastrum integrum Nägeli & & & & & $\mathrm{x}$ & $\mathrm{x}$ \\
\hline Pediastrum sp. & $\mathrm{x}$ & $\mathrm{x}$ & & & & \\
\hline Pseudopediastrum boryanum (Turpin) E.Hegewald & & & $\mathrm{x}$ & & & \\
\hline Scenedesmus incrassatulus Bohlin & & & & & $\mathrm{x}$ & \\
\hline Scenedesmus quadricauda (Turpin) Brébisson & & & $\mathrm{x}$ & $\mathrm{x}$ & & \\
\hline Scenedesmus sp. & $\mathrm{x}$ & $\mathrm{x}$ & $\mathrm{x}$ & $\mathrm{x}$ & & \\
\hline Selenastrum sp. & & $\mathrm{x}$ & & & & \\
\hline Stauridium tetras (Ehrenberg) E.Hegewald & & & $\mathrm{x}$ & & & \\
\hline Volvox sp. & & & $\mathrm{x}$ & & & \\
\hline \multicolumn{7}{|l|}{ DIVISIÓN: EUGLENOPHYTA } \\
\hline Euglena sp. & $\mathrm{x}$ & $\mathrm{x}$ & $\mathrm{x}$ & & & \\
\hline Peranemopsis trichophora (Ehrenberg) L.Péterfi & & & & & $\mathrm{x}$ & \\
\hline Phacus torta (Lemmermann) Skvortsov & & & $\mathrm{x}$ & $\mathrm{x}$ & & \\
\hline Phacus sp. & & $\mathrm{x}$ & & & $\mathrm{x}$ & $\mathrm{x}$ \\
\hline \multicolumn{7}{|l|}{ DIVISIÓN: XANTHOPHYTA } \\
\hline Vacuolaria virescens Cienkowski & & & & & $\mathrm{x}$ & $\mathrm{x}$ \\
\hline
\end{tabular}

\section{Discusión.}

El orden descendente de riqueza específica de los grupos estudiados, tal como se indica a continuación: Bacillariophyta, Chlorophyta, Cyanobacteria, Euglenophyta y Xanthophyta, fue reportado en el mismo sistema por Iannacone et al. (2013) en un estudio de diversidad del plancton y macrozoobentos como indicador alternativo de calidad de agua. La división Bacillariophyta fue la de mayor riqueza específica, patrón presentado en otras investigaciones realizadas en ambientes lóticos (Franco et al., 2003 en
Perú; Martínez et al., 2007 en Argentina; Silva et al., 2008 en Costa Rica; Ribeiro et al., 2009 en Brasil; y Moreno et al., 2008 en México). Según López \& Altamirano (2011), las algas diatomeas constituyen uno de los grupos taxonómicos más abundantes en los sistemas acuáticos, de tal manera que en ríos suponen alrededor del $80-90 \%$ de la comunidad de microorganismos fitoplanctónicos (Rivas et al., 2010). Estas algas son un grupo de organismos con un gran número de especies, vasta distribución por todo el mundo (Soler et al., 2012) y ampliamente 


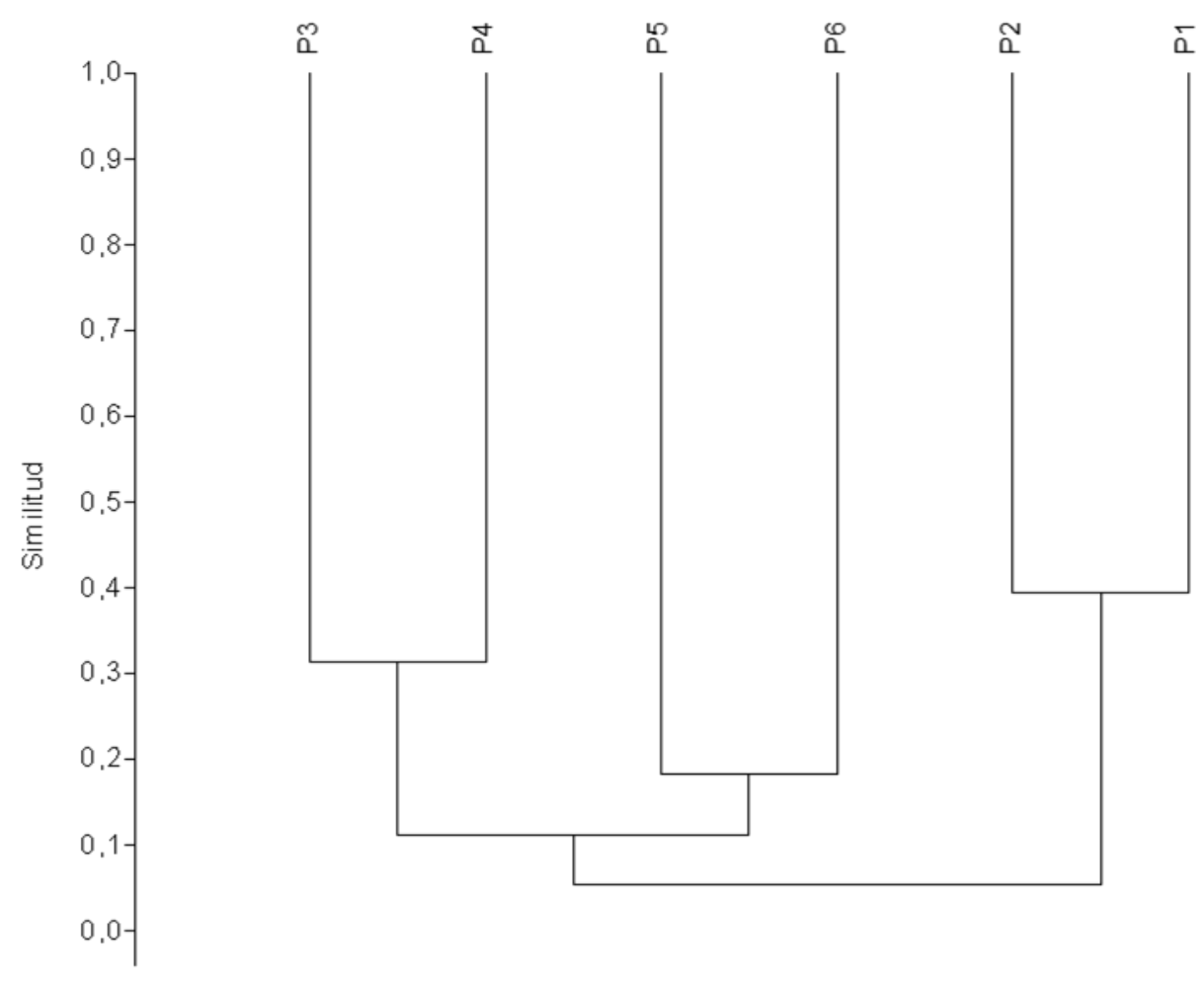

Figura 3. Dendrograma (método UPGMA) generado a partir de una matriz de distancias calculadas con el índice de Jaccard entre seis sitios de muestreo en la cuenca baja de río Lurín, Lima, Perú, en agosto de 2008. P1: Sitio de muestreo 1; P2: Sitio de muestreo 2; P3: Sitio de muestreo 3; P4: Sitio de muestreo 4; P5: Sitio de muestreo 5; P6: Sitio de muestreo 6.

diversificado, ya que existen muchas especies con distintas sensibilidades frente a la contaminación (Ciutti, 2005). La división Bacillariophyta se presentó en todos los sitios de muestreo, pudiendo relacionarse con el aumento de materia orgánica en el curso de agua (Pinilla, 1998). El fenómeno mencionado genera gran influencia en la población de microalgas, aumentando la diversidad, composición y abundancia de diatomeas (Arcos-Pulido \& Gomez-Prieto, 2006; Yucra \& Tapia, 2008), lo que podría explicar el comportamiento de P3, P4, P5 y P6, (menores niveles de similitud) sitios influenciados por la descarga de aguas residuales del proyecto "MESÍAS" SEDAPAL. Peña et al. (2005) mencionan que la riqueza específica puede aumentar o disminuir en respuesta a cambios moderados en la calidad de agua, todo depende si estos representan tensión en las especies; lo que podría estar relacionado con la mayor riqueza especifica en P5 (43) y la menor en P1 (17). El sitio uno se asoció a influencia antropogénica, por tanto, incurre en una modificación de la riqueza específica tal como se ha reportado en un estudio sobre la evaluación de la calidad de agua fluvial con diatomeas en Tacna, Perú (Calizaya-Anco et al., 2013), donde se evidencia una menor diversidad de diatomeas en sitios con características semejantes a
P1. Los valores de similitud registrados entre sitios de muestreo indican que la composición de especies de microalgas y cianobacterias varía a medida que aumenta la distancia espacial entre estos. Esto puede deberse a la influencia de focos contaminantes (Calizaya-Anco et al., 2013) y su distancia a los sitios de muestreo. Así, en los sitios cercanos (rio abajo) de la descarga directa del efluente del proyecto "MESÍAS" (P3 y P4) se presentaron especies tolerantes a la alta polución orgánica tales como Navicula tripunctata (Licursi \& Gómez, 2003) y Oscillatoria sp. (Peralta, 2005). Por otro lado, en los sitios cercanos a la desembocadura (P5 y P6) se registraron tanto especies tolerantes a metales pesados: Gomphonema parvulum y Navicula sp. (Velázquez et al., 2006); como especies indicadoras de eutrofización: Fragilaria crotonensis, Melosira varians, Gomphonema parvulum (Licursi \& Gómez, 2003; Velázquez et al., 2006), entre otros.

Scenedesmus sp. y Stephanodiscus astraea fueron algunas de las especies más frecuentes en los sitios de muestreo. Según Bazán (2010), las especies del género Scenedesmus son de crecimiento rápido y alta tasa de renovación, indicativo de un grado de mineralización de la materia orgánica de moderado a alto. Esto corrobora lo antes mencionado en cuanto al estado de 
Tabla 3. Similitud composicional (índice de Jaccard, $100 \times I_{J}$ ) entre seis sitios de muestreo en la cuenca baja de río Lurín, Lima, Perú, en agosto de 2008. P1: Sitio de muestreo 1; P2: Sitio de muestreo 2; P3: Sitio de muestreo 3; P4: Sitio de muestreo 4; P5: Sitio de muestreo 5; P6: Sitio de muestreo 6.

\begin{tabular}{cccccc}
\multicolumn{7}{c}{ P2 } & P3 & P4 & P5 & P6 \\
\hline P1 & 39,3 & 5,4 & 2,8 & 3,4 & 2,6 \\
P2 & & 12,8 & 7,7 & 3,2 & 4,8 \\
P3 & & & 31,3 & 8,3 & 4,8 \\
P4 & & & & 14,5 & 16,7 \\
P5 & & & & & 18,2 \\
\hline
\end{tabular}

contaminación del sistema. Asimismo, Alhonen (1986) menciona que Stephanodiscus astraea es un claro indicador de desarrollo hacia la eutrofia y polución, lo cual coincide con lo hallado por Cruz \& Ávila (2011) en un estudio de identificación de bioindicadores en la laguna de Zupitlán, México.

Iannacone et al. (2013) describen a Navicula como un género dominante en la cuenca baja del río Lurín, confirmando nuestros resultados. Sin embargo, Vouilloud et al. (2005) y Cuesta et al. (2007) afirman que este género posee una amplia distribución en ambientes dulceacuícolas. Además, Gómez et al. (2001), Bellinger \& Sigee (2010), Segura-García et al. (2012) y Molina (2013) afirman que este género es tolerante a la contaminación y su dominancia indica sitios altamente perturbados, lo que estaría asociado a procesos de contaminación en la cuenca baja del río Lurín.

Las especies $N$. cryptotenella y $N$. reinhardtii se describen como especies sensibles a la contaminación y han sido reportadas en ambientes lóticos en países como Polonia (Rakowska, 2001) y Corea (Lee, 2012). Por otro lado, las especies $N$. accomoda, $N$. cryptocephala, $N$. schroeteri, y $N$. subalpina se describen como tolerantes, como lo afirman Rakowska (2001); Licursi \& Gómez (2003) y Cruz \& Ávila (2011), quienes también destacan su presencia en ecosistemas de aguas corrientes en un estudio sobre diatomeas en el condado de Örebro (Suecia) (Kahlert, 2008); todas las especies mencionadas se presentaron exclusivamente en $\mathrm{P} 5$, lo que podría indicar que la frecuencia vehicular, presencia moderada de residuos sólidos e influencia directa del proyecto "MESÍAS" SEDAPAL estarían perturbando la dinámica natural de este ecosistema.

La temperatura del agua evaluada en los sitios de muestreo concuerda con un Estudio de Línea Base de la Municipalidad Distrital de Cieneguilla (2012), y a su vez coincide con temperaturas indicadas en otras investigaciones que han registrado también especies pertenecientes a la división Bacillariophyta, tales como Cymbella affinis, Diatoma vulgaris (Martínez de Marco \& Tracanna, 2012),
Gomphonema parvulum, Nitzschia palea (Velázquez et al., 2006; Sosa et al., 2011), Melosira varians, Synedra ulna (Sosa et al., 2011), entre otras. Al contrario de lo reportado por Calizaya-Anco et al. (2013) en el río Locumba (Tacna), en el presente estudio la temperatura se redujo gradualmente hasta llegar a la desembocadura, lo que podría estar relacionado con la disminución de áreas urbanas a mayor cercanía al mar; esto debido al incremento de temperatura en la superficie y atmósfera que generan los entornos urbanizados a comparación de los no urbanizados (Voogt, 2008).

En general, los valores de $\mathrm{pH}$ y conductividad eléctrica aumentaron en dirección al mar, coincidiendo con Martínez et al. (2001), quienes hicieron un estudio en la cuenca baja del río Manzanares (Venezuela); además, se observa una relación directa entre ambos parámetros, comportamiento registrado por López et al. (2012). Sin embargo, en nuestra investigación el $\mathrm{pH}$ en el último sitio de muestreo disminuyó de 8.2 a 7.5, lo que podría deberse al aumento de la cercanía al mar (Martínez et al., 2001). Según Morales et al. (2006) las microalgas pueden desarrollarse a un $\mathrm{pH}$ entre 6 y 9, lo que explicaría su presencia en todos los sitios de muestreo. Los autores señalan además, que las diatomeas predominan a valores de $\mathrm{pH}$ ligeramente ácidos, pues hay una mayor disponibilidad de nutrientes y $\mathrm{CO}_{2}$. No obstante, en la presente investigación han sido registradas también a $\mathrm{pH}$ mayores a 7 , lo que supone una competencia para las cianobacterias las cuales pueden estar presentes en este intervalo e incluso a $\mathrm{pH}$ mayores a 9 (Morales et al., 2006); esto explicaría por qué las pocas especies de cianobacterias registradas están presentes como máximo en dos sitios de muestreo cada una. Con respecto a la alta mineralización del sistema, esta es consecuencia de factores naturales $y$ aportes provenientes de los drenajes agrícolas (Municipalidad Distrital de Cieneguilla 2012); su incongruencia frente a valores bajos de $\mathrm{pH}$ en los tres primeros sitios de muestreo puede deberse a una re-suspensión de sedimentos contaminados (Pereira et al., 1995). La mayoría de especies registradas son tolerantes a altos valores de C.E., destacando a Diatoma vulgaris (Martínez de Marco \& Tracanna, 2012), Gomphonema

Tabla 4. Valores de conductividad eléctrica, $\mathrm{pH}$, temperatura y profundidad media del cauce, medidos en seis sitios de muestreo de la cuenca baja del río Lurín, Lima, Perú, en agosto de 2008.

\begin{tabular}{|c|c|c|c|c|}
\hline $\begin{array}{c}\text { Sitio de } \\
\text { muestreo }\end{array}$ & $\begin{array}{l}\text { Conductividad } \\
\text { eléctrica } \\
\left(\mu \mathrm{\mu S} . \mathrm{cm}^{-1}\right)\end{array}$ & pH & $\begin{array}{c}\text { Temperatura } \\
\left({ }^{\circ} \mathrm{C}\right)\end{array}$ & $\begin{array}{c}\text { Profundidad } \\
\text { media del } \\
\text { cauce }(\mathrm{cm})\end{array}$ \\
\hline P1 & 1390 & 6.2 & 23 & 12 \\
\hline $\mathrm{P} 2$ & 1520 & 6.1 & 22 & 33 \\
\hline P3 & 1510 & 6.8 & 21 & 25 \\
\hline P4 & 1410 & 7.2 & 21 & 38 \\
\hline P5 & 1400 & 8.2 & 19 & 12 \\
\hline P6 & 1820 & 7.5 & 16 & 66 \\
\hline
\end{tabular}


parvulum, Oscillatoria sp., Melosira varians y Synedra ulna (Sosa et al., 2011). Lo mismo ocurre con ciertas especies de diatomeas de los géneros Navicula y Nitzschia, tal como lo reportan Calizaya-Anco et al. (2013) en el río Locumba, el cual muestra valores de C.E. incluso más elevados que la actual investigación. La presencia de cianobacterias se explica ya que este grupo puede adaptarse a altas conductividades mediante el desarrollo de adaptaciones fisiológicas (O’Farrell et al., 2003).

\section{Conclusiones.}

La diversidad de microalgas y cianobacterias en cursos de agua es importante para conocer el impacto antropogénico causado por el vertimiento de residuos sólidos y desagües domésticos; algunas especies de los géneros Navicula y Nitzschia dentro del grupo de las Bacillariophyceae confirman esta posición. El aumento de los niveles de recambio con la distancia espacial entre los sitios de muestreo deja clara la sensibilidad de estos organismos a los cambios ambientales como la temperatura del agua y el establecimiento de viviendas; la alta mineralización presente en el sistema debe su origen a factores naturales $\mathrm{y}$ aportes provenientes de los drenajes agrícolas, que se intensifican en los sitios más bajos; la re-suspensión de sedimentos contaminados explica la presencia de valores de $\mathrm{pH}$ bajos; asimismo, la disminución gradual de la temperatura en dirección al mar se explica por la presencia de áreas urbanas alejadas de la desembocadura, las cuales generan islas de calor.

\section{Agradecimientos.}

A Gabriela Sá Leitão, Brenton Ladd, Carlos Acosta, Gustavo Dreyfus y Antonio Abad por sus valiosos comentarios a la presente investigación.

\section{Literatura citada.}

Acleto C. \& Zúñiga R. 1998. Introducción a las algas. Lima, Perú: Escuela Nueva.

Alfaro J.C. \& Claverías R. 2010. Diagnóstico socioeconómico de la cuenca del río Lurín [Consultoría sobre Sistema de Monitoreo y Evaluación, Línea de Base y Mapa de Actores]. Lima, Perú: Centro Global para el Desarrollo y la Democracia (CGDD), Centro de Investigación, Educación y Desarrollo (CIED).

Alhonen P. 1986. Holocene lacustrine microfossils and environmental changes. Bulletin of the Geological Society of Finland. 58: 57-69.

Alvariño L. \& Iannacone J. 2007. Diversidad de Invertebrados Acuáticos de la Bocatoma de la Atarjea en el Río Rímac, Lima Perú durante 1999. Biotempo. 7: 6175.

Arcos-Pulido M.P. \& Gómez-Prieto A.C. 2006. Microalgas perifíticas como indicadoras del estado de las aguas de un humedal urbano Jaboque, Bogotá D.C., Colombia. NOVA Publicación Científica. 4: 60-79.

Bazán G.I. 2010. Estudios ficológicos en el sistema léntico de La Arocena (Departamento Maracó), La Pampa,
Argentina (Tesis Doctoral). Universidad Nacional del Río Cuarto (UNRC), Río Cuarto, Argentina.

Bellinger E.G. \& Sigee D.C. 2010. Freshwater algae Identification and use as bioindicators. Oxford: WileyBlackwell.

Bicudo C.E.D.M. \& Menezes M. 2006. Gêneros de Algas de Águas Continentais do Brasil (chave para identificação e descrições). São Carlos, Brasil: RiMa.

Borcard D., Gillet F. \& Legendre P. 2011. Numerical Ecology with R. Use R! series. New York: Springer.

Calizaya-Anco J., Avendaño-Cáceres M. \& Delgado-Vargas I. 2013. Evaluación de la calidad del agua fluvial con diatomeas (Bacillariophyceae), una experiencia en Tacna, Perú. Revista Peruana de Medicina Experimental y Salud Pública. 30: 58-63.

Ciutti F. 2005. Il monitoraggio dei corsi d'acqua con indicatori algali (diatomee). Annali dell'Istituto Superiore di Sanità. 41: 393-397.

Coral F. 2007. Zonificación Mediante Planos Temáticos de Áreas con Diferente Peligro en la Parte Baja del Valle de Lurín (Tesis para optar el Título de Ingeniero Civil). Universidad Ricardo Palma, Lima, Perú.

Cruz M. \& Ávila A. 2011. Identificación de bioindicadores en la laguna de Zupitlán, Municipio de Acatlán, Hidalgo, México. En "III Encuentro de Investigación del Área Académica de Ciencias de la Tierra y Materiales”. Hidalgo, México: Universidad Autónoma del Estado de Hidalgo.

Cuesta J.E.L., Olofsson L. \& Sánchez-Castillo P. 2007. Comunidades de diatomeas epipélicas en las lagunas de alta montaña de Sierra Nevada (Granada, España). Limnética. 26: 99-113.

Felipe-Morales C. 2012. Manual para Gestores del Agua de la Cuenca del Río Lurín [Proyecto Desarrollo Territorial y Generación de Empleo en la Cuenca del Río Lurín]. Lima, Perú: Centro Global para el Desarrollo y la Democracia (CGDD), Centro de Investigación, Educación y Desarrollo (CIED), Fondo Multilateral de Inversiones (FOMIN-BID).

Franco J., Sulca L. \& Cáceres C. 2003. Fitoplancton del río altoandino "Kaño" - Tacna (Cordillera suroccidental del Perú). Ciencia \& Desarrollo. 7: 49-55.

Galati B.G., Monacci F., Gotelli M.M. \& Rosenfeldt S. 2007. Pollen, tapetum and orbicule development in Modiolastrum malvifolium (Malvaceae). Annals of Botany. 99: 755-763.

Gómez L., Larduet Y. \& Abrahantes N. 2001. Contaminación y biodiversidad en ecosistemas acuáticos. El fitoplancton de la bahía de Santiago de Cuba. Revista de Investigaciones Marinas. 22: 191-197.

Iannacone J., Alvariño L., Jiménez-Reyes R. \& Argota G. 2013. Diversidad del plancton y macrozoobentos como indicador alternativo de calidad de agua del río Lurín en el distrito de Cieneguilla, Lima-Perú. The Biologist (Lima). 11: 79-95.

Iannacone J., Alvariño L., Moreno R., Reyes M. \& Chauca J. 2000. Culícidos (Diptera) del río Chillón y sectores adyacentes de la provincia constitucional del Callao, Perú, durante el Niño 1997-1998. Acta entomológica chilena. 24: 51-60.

Kahlert M. 2008. Kiselalgsundersökning i södra delen av Norra Östersjödistriktet, 2007. Departament of Environmental Assessment, Swedish University of Agricultural Sciences, Sweden. 
Komárek J. \& Anagnostidis K. 1998. Cyanoprokaryota. 1. Chroococcales. En H. Ettl, G. Gärtner, H. Heynig \& D. Mollenhauer (eds.). Süßwasserflora von Mitteleuropa (19/1). Gustav Fischer, Jena- Stuttgart-Lübeck-Ulm.

Komárek J. \& Anagnostidis K. 2005. Cyanoprokaryota. 2. Oscillatoriales. En: B. Büdel, L. Krienitz, G. Gärtner \& M. Schagerl (eds.). Süßwasserflora von Mitteleuropa (19/2). Heidelberg: Elsevier/Spektrum.

Kshirsagar A.D. 2013. Bioremediation of wastewater by using microalgae: an experimental study. International Journal of Life Science Biotechnology and Pharma Research. 2(3): 339-346.

Lee J.H. 2012. Algal Flora of Korea. Flora and Fauna of Korea (vol. 3, núm. 10). Chrysophyta: Bacillariophyceae: Pennales: Raphidineae: Naviculaceae: 20 genera including Navicula. Incheon: National Institute of Biological Resources.

Licursi M. \& Gómez N. 2003. Aplicación de índices bióticos en la evaluación de la calidad del agua en sistemas lóticos de la llanura Pampeana Argentina a partir del empleo de diatomeas. Biología Acuática. 21: 31-49.

Lobo E.A., Callegaro V.L.M., Hermany G., Bes D., Wetzel C.A. \& Oliveira M.A. 2004. Use of epilithic diatoms as bioindicators for lotic systems in southern Brazil, with special emphasis on eutrophication. Acta Limnologica Brasiliensia. 16: 25-40.

Lobo E.A., Katoh K. \& Aruga Y. 1995. Response of epilithic diatom assemblages to water pollution in rivers in the Tokyo Metropolitan área, Japan. Freshwater Biology. 34: 191-204.

López F.O. \& Altamirano M.C. 2011. Diatomeas bentónicas de los oasis de Baja California Sur [Informe Final SNIBCONABIO, Proyecto No. HJ032]. La Paz, México D.F.: Universidad Autónoma de Baja California Sur.

López M., Monks S., Serrano A., Pulido G., Gaytan J.C. \& López M. 2012. Dinámica de las variables físicoquímicas del sedimento de la laguna Tampamachoco, Veracruz, México. Revista científica de la escuela de Ingeniería Agronómica de la Universidad del Oriente. 12: 965 - 972 .

Martínez A.L., Luque M.E., Lombardo D. \& Bruno E. 2007. Potamoplancton en la cuenca media del río Cuarto (Córdoba, Argentina). Limnetica. 26: 25-38.

Martínez G., Alvarado J. \& Señor W. 2001. Estudio físicoquímico de las aguas superficiales de la cuenca baja y pluma del río Manzanares. Interciencia. 26: 342-351.

Martínez de Marco S.N. \& Tracanna B.C. 2012. Variaciones temporales del fitoplancton de los ríos tributarios y emisarios del embalse C. Gelsi (Tucumán, Argentina). Boletín Sociedad Argentina de Botánica. 47: 323-340.

Medina J., Piña V., Nieves S., Arzola G. \& Guerrero I. 2012. La importancia de las microalgas. CONABIO. Biodiversitas. 103: 1-5.

Meybeck M., Friedrich G., Thomas R. \& Chapman D. 1992. Rivers. En D. Chapman (ed.). Water Quality Assessments: a guide to use of biota, sediments and water in environmental monitoring (pp. 239-316). Londres: Chapman \& Hall.

Ministerio de Agricultura del Perú. 2004. Estudio Hidrológico de la cuenca del río Lurín [Informe Final]. Lima, Perú: Administración Técnica del Distrito de Riego Chillón - Rímac - Lurín.
Molina M. 2013. Efectos del derrame de petróleo sobre la comunidad fitoplanctónica de la laguna de Papallacta y sus principales afluentes (Tesis de Licenciatura). Universidad internacional del Ecuador, Quito, Ecuador.

Morales N., Arévalo K., Ortega J., Briceño B., Andrade C. \& Morales E. 2006. El pH y la fuente nitrogenada como moduladores del crecimiento de la macrófita Lemna sp. Revista de la Facultad de Agronomía (LUZ). 23: 67-79.

Moreno J.L., Tapia M., González M.C. \& Figueroa M.G. 2008. Fitoplancton del río Tehuantepec, Oaxaca, México, y algunas relaciones biogeográficas. Revista de biología tropical. 56: 27-54.

Municipalidad Distrital de Cieneguilla. 2012. Plan de Desarrollo Local Concertado PDLC 2012 - 2021 [Estudio de Línea Base - Estudio de Medición]. Lima: Autor.

O’Farrell I., Sinistro R., Izaguirre I. \& Unrein F. 2003. Do steady state assemblages occur in shallow lentic environments from wetlands?. Hydrobiologia. 502: 197209.

Peña E.J., Palacios M.L. \& Ospina-Álvarez N. 2005. Algas como indicadoras de contaminación. Cali: Universidad del Valle.

Peralta P. 2005. Fitobentos, fitoplancton y zooplancton del Litoral del Bañado de Carilauquen, Cuenca de llancanelo, Mendoza, Argentina. Limnetica. 24(1-2): 183-198.

Pereira E., Moura Y., Costa J., Mahony J. \& Toman R. 1995. The Sto. Domingo mine: A study of heavy metals contamination in the water column and sediments of the Chanca River Basin by discharge from an ancient cupriferous pyrite mine (Portugal). Marine and Freshwater Research. 46: 145-151.

Pinilla G.A. 1998. Indicadores biológicos en ecosistemas acuáticos continentales de Colombia. Compilación bibliográfica. Colombia: Fundación Universidad de Bogotá Jorge Tadeo Lozano.

Rakowska B. 2001. Study of diatom diversity in water ecosystems of Poland's lowlands. Ecohydrology \& Hydrobiology. 1(4): 481-502.

Ribeiro M.D., Alves N.F., Moreno da Silva M.A. \& Santos R. 2009. Composição e distribuição do microfitoplâncton do rio Guamá no trecho entre Belém e São Miguel do Guamá, Pará, Brasil. 0. 4(3): 341-351.

Rivas A.W., Gómez R.E. \& Monterrosa A.J. 2010. Consideraciones generales para el estudio y monitoreo de diatomeas en los principales ríos de El Salvador. En Sermeño, J.M. \& M. Springer (eds.). Formulación de una guía metodológica estandarizada para determinar la calidad ambiental de las aguas de los ríos de El Salvador, utilizando insectos acuáticos [Proyecto Universidad de El Salvador (UES) - Organización de los Estados Americanos (OEA)]. San Salvador: Universidad de El Salvador.

Roset J., Aguayo S. \& Muñoz M.J. 2001. Detección de cianobacterias y sus toxinas. Una revisión. Revista de Toxicología. 18: 65-71.

Ruiz J., Junes K. \& La Torre M.I. 2007. Flora algal del río Chillón en la provincia de Lima, Perú. The Biologist (Lima). 5(2): 43-51.

Segura-García V., Cantoral-Uriza E.A., Israde I. \& Maidana, N. 2012. Diatomeas epilíticas como indicadores de la calidad del agua en la cuenca alta del río Lerma, México. Hidrobiológica. 22: 16-27. 
Silva A.M., Sili C. \& Torzillo G. 2008. Cyanoprocaryota y microalgas (Chlorophyceae y Bacillariophyceae) bentónicas dominantes en ríos de Costa Rica. Revista de biología tropical. 56: 221-235.

Soler A., Pérez M.I., Aguilar E. \& Villarreal I. 2012. Diatomeas del Canal de Panamá: Bioindicadores y otros estudios pioneros. Panamá: Universidad de Panamá.

Sosa M.L., Novoa M.D. \& Martínez de Fabricius A.L. 2011 Ficoflora de la cuenca endorreica fluviolacustre Chucul (Córdoba, Argentina). Biológicas. 13(1): 14-23.

Vázquez G., Castro G., Gonzalez G., Pérez R. \& Castro T. 2006. Bioindicadores como herramientas para determinar la calidad del agua. Contactos. 60: 41-48.

Vega G.A. 2010. The rural agribusiness development and environmental conservation in highland areas of Peru (Tesis de Maestría en Ciencias). Industrial Ecology Royal Institute of Technology, Estocolmo, Suecia.

Velázquez M.M., Israde I. \& Mendoza M. 2006. Uso de diatomeas para la evaluación de la calidad del agua del río Turbio, afluente del río Lerma, México. En Simposio "Ecología y Manejo de Ecosistemas Acuáticos en
Michoacán”. Congreso Mexicano de Ecología, Morelia, Michoacán, México.

Vélez-Azañero A. \& Lizárraga-Travaglini A. 2013. Diversidad de Carabidae (Coleoptera) asociados a la cuenca baja del río Lurín, Lima, Perú. The Biologist (Lima). 11: 97-106.

Voogt J.A. 2008. Islas de Calor en Zonas Urbanas: Ciudades Más Calientes. EEUU: American Institute of Biological Sciences.

Vouilloud A.A., Sala S.E. \& Sabbatini M.R. 2005. Diatomeas perifíticas de la cuenca del Río Sauce Grande (Buenos Aires, Argentina). Iheringia. 60: 77-89.

Wan Maznah W.O. 2010. Perspectives on the Use of Algae as Biological Indicators for Monitoring and Protecting Aquatic Environments, with Special Reference to Malaysian Freshwater Ecosystems. Tropical Life Sciences Research. 21(2): 51-67.

Yucra H.A. \& Tapia P.M. 2008. El uso de microalgas como bioindicadoras de polución orgánica en Brisas de Oquendo, Callao, Perú. The Biologist (Lima). 6: 41-47.

\footnotetext{
${ }^{1}$ Universidad Científica del Sur. Facultad de Ciencias Ambientales. Carrera de Ingeniería Ambiental. Panamericana Sur km 19. Lima 42. Correo electrónico: fonia14@gmail.com
} 